\title{
Decreased Joint Pain Associated with Curcumin Use in a Controlled Study for Alzheimer's Disease: A Serendipitous Observation
}

\author{
John M Ringman ${ }^{1}$, Greg M Cole ${ }^{1,2}$ and Sally Frautschy*1,2 \\ ${ }^{1}$ Department of Neurology, Mary S Easton Center for Alzheimer's Disease Research, USA \\ ${ }^{2}$ Department of Neurology, Geriatric Research Education and Clinical Center, USA
}

*Corresponding author: Sally Frautschy, Geriatric Research Education and Clinical Center, WLA Veterans Administration Medical Center, USA.

Received Date: January 06, 2020

Published Date: January 29, 2020

\begin{abstract}
Curcumin is a natural product with anti-inflammatory properties for which there is preliminary evidence of efficacy in the treatment of arthritis. We performed a 24-week, randomized, placebo-controlled, and blinded study of curcumin for Alzheimer's disease in which there was a 24-week open-label extension. At each visit, subjects and their informants were specifically queried about adverse events with a comprehensive checklist that included the presence or absence of joint pain. We serendipitously found a dose-dependent trend for decreased reports of joint pain. This observation provides evidence for the efficacy of curcumin for the treatment of joint pain in the elderly.
\end{abstract}

\section{Key Message}

In a trial of curcumin for Alzheimer's disease, we serendipitously observed a dose-dependent trend for decreased complaints of joint pain.

Age-associated medical conditions are growing public health care problems for which safe and effective interventions are sorely needed. Radiographic evidence of knee osteoarthritis increases from $27 \%$ in persons under 70 to $44 \%$ of persons 80 years of age and above [1]. The first-line treatments for various forms of arthritis, the non-steroidal anti-inflammatory drugs, or NSAIDs, can have substantial gastrointestinal and other morbidity associated with them. Many other compounds, including natural products, have anti-inflammatory properties and objective and controlled evidence for their efficacy in various forms of arthritis is growing. Curcumin is a polyphenolic compound derived from the plant Curcuma Long Lin that has been demonstrated to have anti-inflammatory and antioxidant properties as well as efficacy against the pathology of Alzheimer's disease (AD) in animal models [2-4]. Curcumin inhibits lipoxygenase and cyclooxygenase- 2 that synthesize pro- inflammatory leukotrienes, prostaglandins, and thromboxane, blocks cytokine- mediated NF-kappa B activation and proinflammatory gene expression by inhibiting inhibitory factor I-kappa B kinase activity and suppresses inducible nitric oxide synthase in activated macrophages, processes that promote inflammation [5]. Here we report evidence for efficacy of Curcumin in the treatment of joint pain observed serendipitously in a randomized placebo-controlled trial for Alzheimer's disease (AD).

In a preliminary study of the efficacy of curcumin against $\mathrm{AD}$ pathology using plasma and cerebrospinal fluid (CSF) biomarkers, we randomized 36 subjects with mild-to-moderate AD in a placebocontrolled trial of 2 grams and 4 grams of Curcumin C3 Complex. After 24 weeks, persons receiving placebo were again randomized to receive either 2 or 4 grams of curcumin (ClinicalTrials.gov Identifier: NCT00099710.) [6]. Exclusionary medications included aspirin at doses greater than $325 \mathrm{mg} /$ day, use of NSAIDs more than three times per week and the use of antioxidant supplements (for example, coenzyme Q10 and alpha-lipoic acid). At baseline and each of the post-baseline visits (weeks 4, 12, 24, 36, and 48) subjects and caregivers were interviewed regarding adverse events using an extensive checklist in which the presence or absence of 78 symptoms was queried. Among the symptoms systematically inquired about at each visit was the presence or absence of "joint 
pain". At screening and at each post-baseline visit laboratory value monitoring was also performed. Occurrence of adverse events per subject according to the treatment they were receiving was compared between treatment groups for all subjects using the general linear mixed model.

The mean age of subjects was 74 years. Thirty subjects completed the 24-week blinded phase and 28 completed the full 48-week study. Seven people dropped out while on curcumin of which four were due to gastrointestinal side effects. Joint pain was reported at $15 \%$ of visits for persons on placebo, $9 \%$ of visits for persons on $2 \mathrm{gm}$ and $5 \%$ of persons on $4 \mathrm{gm}$ of curcumin $(\mathrm{p}=$ 0.19). When modeling the difference in joint pain occurrence as a linear function of curcumin dose, a $2.5 \%$ trend towards decrease in joint pain per gram of curcumin was found $(\mathrm{p}=0.07)$. This apparent dose-dependent effect was not seen with any of the 77 other symptoms that were queried. There were no differences in levels of C-reactive protein, erythrocyte sedimentation rate, or F2isoprostanes between treatment groups.

We were unable to demonstrate an effect of curcumin on biomarkers for AD in our pilot study. Though plasma levels of native curcumin and its metabolite tetrahydro curcumin were low $(<10$ $\mathrm{ng} / \mathrm{mL}$ ), mean levels of glucuronidated curcumin and tetrahydro curcumin were 96 and $298 \mathrm{ng} / \mathrm{mL}$ respectively. It is possible that unmeasured metabolites of curcumin or their glucuronidated forms that do not penetrate the central nervous system might nonetheless have peripheral anti-inflammatory effects.

An ameliorative effect of curcumin has been found in relatively small randomized blinded clinical trials for both osteoarthritis Panahi Y et al. [7] and rheumatoid arthritis [8]. Though our study was not designed to detect an effect on arthritic symptoms, our findings, though not statistically significant, of a trend for a dosedependent decrement in the prevalence of joint pain complaints is additional supportive evidence for a clinically relevant anti- inflammatory effect of curcumin for such symptoms in the elderly. These serendipitous results support further study of preparations of curcumin with substantiated bioavailability for the treatment of inflammatory disorders.

\section{Acknowledgement}

None.

\section{Conflicts of Interest}

No conflict of interest.

\section{References}

1. Felson DT, Naimark A, Anderson J, Kazis L, Castelli W, et al. (1987) The prevalence of knee osteoarthritis in the elderly. The Framingham Osteoarthritis Study. Arthritis Rheum 30(8): 914-918.

2. Lim GP, Chu T, Yang F, Beech W, Frautschy SA, et al. (2001) The curry spice curcumin reduces oxidative damage and amyloid pathology in an Alzheimer transgenic mouse. J Neurosci 21(21): 8370-8377.

3. Ma QL, Zuo X, Yang F, Ubeda OJ, Gant DJ, et al. (2013) Curcumin suppresses soluble tau dimers and corrects molecular chaperone, synaptic, and behavioral deficits in aged human tau transgenic mice. J Biol Chem 288(6): 4056-4065.

4. Garcia Alloza M, Borrelli LA, Rozkalne A, Hyman BT, Bacskai BJ (2007) Curcumin labels amyloid pathology in vivo, disrupts existing plaques, and partially restores distorted neurites in an Alzheimer mouse model. J Neurochem 102(4): 1095-1104.

5. Aggarwal BB, Sung B (2009) Pharmacological basis for the role of curcumin in chronic diseases: an age-old spice with modern targets. Trends Pharmacol Sci 30(2): 85-94.

6. Ringman JM, Frautschy SA, Teng E, Begum AN, Bardens J, et al. (2012) Oral curcumin for Alzheimer's disease: tolerability and efficacy in a 24week randomized, double blind, placebo- controlled study. Alzheimers Res Ther 4(5): 43 .

7. Panahi Y, Rahimnia AR, Sharafi M, Alishiri G, Saburi A, et al. (2014) Curcuminoid Treatment for Knee Osteoarthritis: A Randomized DoubleBlind Placebo-Controlled Trial. Phytother Res 28(11): 1625-1631.

8. Chandran B, Goel A (2012) A randomized, pilot study to assess the efficacy and safety of curcumin in patients with active rheumatoid arthritis. Phytother Res 26(11): 1719-1725. 